\title{
Digitales Marketing kundenzentriert umsetzen
}

Dieses letzte Kapitel beschäftigt sich mit der Digitalisierung des Vertriebskanals und im Besonderen mit den damit verbundenen Auswirkungen auf die Kundenbeziehung. Die Beiträge beleuchten die Einsatzmöglichkeiten neuer Vertriebstechnologien gezielt aus der Sichtweise der potenziellen Konsumenten und ermöglichen somit eine Bewertung der Potenziale und Anwendungsszenarien. Die Digitalisierung stellt den Einzelhandel vor große Herausforderungen. Zunehmend kommen Kunden auf mehreren Endgeräten und in mehreren digitalen sowie physischen Kundenkontaktpunkten mit dem Unternehmen in Berührung. Im ersten Beitrag von Hohlbaum et al. werden die Effekte von Virtual Reality auf das wahrgenommene Risiko im Einkaufsprozess untersucht. Die Ergebnisse der Studie liefern hierbei wertvolle Erkenntnisse für die Einsatzmöglichkeiten dieser Technologie im Einzelhandel. Der zweite Beitrag von Miorin, Braun und Bäumer beschäftigt sich mit der Orchestrierung mehrkanaliger Vertriebsaktivitäten und vertieft insbesondere Anwendungsbeispiele aus dem Einzelhandel. Im nächsten Beitrag von Salomon und Müller wird untersucht wie Kommunikation optimal gestaltet werden kann um die Akzeptanz von vernetzten Haustechnik-Produkten sicherzustellen. Die Digitalisierung ermöglicht auch völlig neue Kundenkontaktprozesse. Chatbots eröffnen die Möglichkeit einer vollständig automatisierten, bilateralen Kommunikation zwischen Kunden und Unternehmen. Ob und unter welchen Bedingungen Kunden dies jedoch akzeptieren, wird im folgenden Abschnitt von Völkle und Planing in verschiedenen Einsatzszenarien untersucht. Abschließend werden hieraus Handlungsempfehlungen abgeleitet.

(C) Springer Fachmedien Wiesbaden GmbH, ein Teil von Springer Nature 2019

A. Lochmahr, P. Müller, P. Planing, T. Popović, Digitalen Wandel gestalten

https.//doi.org/10.1007/978-3-658-24651-8_6.0 